\title{
Evaluation of Anti-SARS-CoV-2 IgG Antibody in Healthcare Professionals Infected with COVID-19
}

\author{
Borhan Moradveisi (iD) ${ }^{1}$, Shirin Behzadi (iD) ${ }^{2}$, Farima Zakaryaei (iD) ${ }^{3}$, Ali Jalili (iD) ${ }^{1}$, Khaled Rahmani (i) ${ }^{4}$ and \\ Avat Karimi (iD 2, * \\ ${ }^{1}$ Cancer and Immunology Research Center, Research Institute for Health Development, Kurdistan University of Medical Sciences, Sanandaj, Iran \\ ${ }^{2}$ Department of Pediatrics, Kurdistan University of Medical Sciences, Sanandaj, Iran \\ ${ }^{3}$ Faculty of Medicine,Kurdistan University of Medical Sciences, Sanandaj, Iran \\ ${ }^{4}$ Liver and Digestive Research Center, Research Institute for Health Development, Kurdistan University of Medical Sciences, Sanandaj, Iran \\ "Corresponding author: Department of Pediatrics, Kurdistan University of Medical Sciences, Sanandaj, Iran. Email: avatkarimi2018@gmail.com
}

Received 2021 October 04; Revised 2021 November 27; Accepted 2021 December 01.

\begin{abstract}
Background: The knowledge of antibody's significance and frequency in patients cured of severe acute respiratory syndrome coronavirus 2 (SARS-CoV-2) is extremely limited.

Objectives: This study aimed to evaluate anti-SARS-CoV-2 IgG antibodies in patients exposed to SARS-CoV-2.

Methods: Healthcare professionals infected with SARS-CoV-2 were enrolled in this study. The levels of anti-SARS-CoV-2 IgG antibodies were detected 15 days after the onset of symptoms and five months later.

Results: A total of 167 patients with coronavirus disease 2019 (COVID-19) were evaluated, including 119 (71.3\%) females and 48 (28.7\%) males. Of the 88 polymerase chain reaction (PCR)-positive patients, 55 (62.5\%) had IgG-positive antibodies, and of the 79 reverse transcriptase (RT)-PCR-negative patients, 12 (16.9\%) had IgG-positive antibodies. Out of 23 anosmia cases, 19 (82.6\%) had positive antibodies. There was a significant relationship between anosmia and positive antibody $(\mathrm{P}=0.001)$, but there was no correlation between antibody titers and gender and other disease symptoms. Immortally, 63 (94\%) cases demonstrated high levels of anti-SARSCoV-2 IgG antibodies after five months of infection. Moreover, 6.5\% $(\mathrm{N}=11)$ of the total population were re-infected with COVID-19 six months later.

Conclusions: Overall, anti-SARS-CoV-2 IgG antibodies detection may be an appropriate method to identify suspected patients with a negative RT-PCR test. Antibodies can remain high in most infected patients for up to five months after infection. Moreover, anosmia seems to be a valuable diagnostic factor, and the healthcare system should implement isolation measures for patients with anosmia.
\end{abstract}

Keywords: Anti-SARS-CoV-2 IgG, COVID-19, Anosmia, RT-PCR

\section{Background}

In late 2019, viral pneumonia cases of unknown cause were reported in Wuhan, China. This new viral disease was called coronavirus disease 2019 (COVID-19) viral pneumonia. The causative agent of COVID-19 is a single-stranded RNA virus. Comparison of the genetic sequence of this virus and other viruses has shown similarities with the severe acute respiratory syndrome (SARS) virus and bat coronavirus. The viruses transmit to humans through an intermediate host $(1,2)$. Human-to-human transmission of the virus has been confirmed. Coronaviruses are transmitted primarily in close contact with COVID-19 patients, mainly through respiratory droplets caused by coughing and sneezing up to about $4 \mathrm{~m}$; it is also found in fecal samples from infected patients. Studies have shown that the virus can be transmitted even during incubation. If left unchecked, the virus typically causes 1.4 to 3.9 new cases per infection. Preliminary estimates indicate that the virus mortality rate is between $1.32 \%$ and $2.04 \%$ of those infected (3-5).

Quantitative reverse transcription-polymerase chain reaction (RT-PCR) was used to analyze samples taken from the upper and lower respiratory tracts for clinical diagnosis of COVID-19; however, it needs special equipment and is time-consuming and costly. Also, since quantitative RTPCR needs samples from the upper and lower respiratory tracts, the process of collecting samples and extracting RNA increases the risk of exposure to viral droplets for personnel. Therefore, an alternative diagnostic test for quantitative RT-PCR seems necessary to identify COVID-19 infection (6)

Serological tests for antibody detection are helpful di- 
agnostic tools widely used to diagnose viruses. These antibodies in the peripheral blood indicate that the person has been exposed to the virus and developed antibodies against it. Naturally, this event protects against reinfection; however, in the case of COVID-19, it is not yet clear how strong the acquired immunity can be or even how long it will last (7). Recent studies have evaluated the levels of anti-severe acute respiratory syndrome coronavirus 2 (SARS-CoV-2) IgG and IgM in the sera of patients with history of SARS-CoV-2. Many reports have shown that IgM and IgG levels increase in the first week of infection (810). Many Iranians, particularly healthcare professionals, have been infected with this virus, like other nationalities. However, only a few studies have followed up these antibodies' levels for a long time after infection.

\section{Objectives}

Herein, we report the importance of anti-SARS-CoV-2 IgG as a functional laboratory test and anosmia as clinical observation for diagnosing COVID-19.

\section{Methods}

\subsection{Patients and Sampling}

A total of 167 healthcare professionals with COVID-19 working at Besat Hospital, Kurdistan University of Medical Sciences (KUMS), were enrolled in this study from March to June 2020.

Inclusion criteria: The study included: (1) patients with confirmed COVID-19 based on quantitative RT-PCR and (2) Patients with confirmed COVID-19 based on Vertical artifacts/pleural irregularities/white lung areas or sub-pleural consolidations on lung Computed Tomography (CT) scan. Exclusion criteria: They were: (1) Death of patients, (2) Reluctance of patients for cooperating and participating in the research, and (3) Not showing up for check-ups and not compliance with therapy for any reason. Three milliliters of venous blood were collected from each of the subjects 15 days and five months after infection. The blood samples were centrifuged at 1,000 rpm for $5 \mathrm{~min}$. To determine the level of IgG in the blood, we isolated serum samples, stored them at a temperature below $20^{\circ} \mathrm{C}$, and then sent them to the laboratory in less than $24 \mathrm{~h}$. Next, IgG levels were measured using the E200519AY Euro Immune Kit (Germany). The sensitivity and specificity of IgG levels for coronavirus detection were $63.3 \%$ and $100 \%$, respectively.

\subsection{Determination of anti-SARS-CoV-2 IgG}

According to the manufacturer's instructions, the levels of anti-SARS-CoV-2 IgG were determined by ELISA (EUROIMMUN, Lübeck, Germany). Briefly, first, $100 \mu \mathrm{L}$ of sample dilution solution was added to all wells. Then, $50 \mu \mathrm{L}$ of controls and samples were poured into the wells according to the instructions; the first two wells were considered blank, and the subsequent two wells were negative controls. Next, the positive control was duplicated, and other wells were used for the samples. The plate was gently shaken for $30 \mathrm{~s}$ until the samples and controls were well mixed with the diluent solution. After covering the plate label walls, the wells were placed at $37{ }^{\circ} \mathrm{C}$ for $30 \mathrm{~min}$. The wells were washed five times with $300 \mu \mathrm{L}$ of ready-to-use washing solution. Then, $100 \mu \mathrm{L}$ of the ready-to-use enzymeconjugated solution was added into the wells, except for the blank wells. The wells were rewashed five times, and $100 \mu \mathrm{L}$ of dye solution was added to all wells. The plate was placed at room temperature in the dark for $15 \mathrm{~min}$. Enzyme reactions stopped by adding $100 \mu \mathrm{L}$ of stop solution to each well.

\subsection{Statistical Analysis}

We used SPSS 21 (SPSS Inc., Chicago, Ill., USA) to analyze the study data, and a P-value of less than 0.05 was considered significant.

\section{Results}

A total of 167 patients with COVID-19 were evaluated, including 119 (71.3\%) females and 48 (28.7\%) males. The disease prevalence was higher in women than in men. The most common symptom in these patients was fever (97\%). Other symptoms included cough and respiratory distress (84\%), myalgia (77\%), weakness (73\%), sore throat (71\%), anosmia and headache (23\%), ageusia (20\%), diarrhea $(8.4 \%)$, and vomiting (7.2\%). Further, 3.0\% of the patients had a history of an underlying disease. In $8.4 \%$ of the patients, pulmonary manifestations of COVID-19 were observed in CT scans. Seventeen patients were hospitalized, none of whom died. Of the 88 PCR-positive patients, 55 (62.5\%) had IgG-positive antibodies, and 33 had negative IgG titers; further, of the 79 PCR-negative patients, 12 (16.9\%) were IgGpositive.

Out of 23 anosmia cases, 19 (82.6\%) had a positive antibody, and there was a significant relationship between anosmia symptom and positive antibody $(\mathrm{P}=0.001)$; however, there was no correlation between antibody titers and gender and other symptoms (Table 1). At the second followup five months later, of the 67 patients with a positive antibody, 63 (94\%) had a positive antibody, and only four (6\%) 
had a negative antibody. The primary symptoms of patients with positive IgG were evaluated, showing that the most common primary symptom was fever (86.6\%), followed by myalgia and weakness (14.9\%), cough and distress (9\%), and headache (3\%); vomiting and diarrhea were not observed in any of the patients. At follow-up, 6.5\% ( $\mathrm{N}=11)$ of the total population were re-infected with COVID-19 six months later; all had positive PCR in the first episode of the disease.

\section{Discussion}

Although COVID-19 was discovered more than a year ago, the role of antibodies against antigens of the virus has not yet been well-documented. Accordingly, we studied the levels of anti-SARS-CoV-2 IgG in healthcare professionals during the disease course and five months after infection. According to the study results, COVID-19 was more common in women, and fever was the most common symptom. Among COVID-19 patients with clinical evidence and lung manifestations, the PCR test was negative in about half of the patients. Also, the present study showed that the IgG antibody titer was high in a small minority of PCR-negative patients. Therefore, it can be suggested that the PCR test has a high false-negative level; accordingly, the anti-SARS-CoV-2 IgG test may be an appropriate method to identify suspected patients with a negative PCR test. These results are in line with a recent report showing that the detection of anti-SARS-CoV-2 IgG and IgM can help diagnose asymptomatic infections and suspected patients with negative RT-PCR results (11).

Adams et al. (9) examined the anti-SARS-CoV-2 IgG and IgM levels in patients with a history of SARS-CoV-2. The study was performed on 40 plasma samples of RT-PCRpositive patients. Also, 142 plasma samples collected before the outbreak of COVID-19 (before December 2019) were used as controls. The IgM and IgG ELISA results were positive in 34 of 40 patients with a previous diagnosis of COVID19 (sensitivity of $85 \%$ and specificity of $95 \%$ ). The IgG titers were also positive in 31 of 31 patients more than 10 days after the onset of symptoms. Anti-SARS-CoV-2 IgG titers increased three weeks after symptom onset and dropped eight weeks later. They showed that IgM and IgG 10 days after symptom onset were specific for diagnosing COVID-19 infection (9).

In 2020, Long et al. (11) examined IgM and IgG antibody levels in patients with a history of SARS-CoV-2. The study was performed on 285 COVID-19 patients. Nineteen days after the onset of COVID-19 symptoms, the IgG test was positive in all patients. IgG peaked around 17 - 19 days after the onset of symptoms, and IgM peaked around 2022 days. Also, in the first three weeks after the onset of symptoms, the titers of anti-SARS-CoV-2 IgG and IgM antibodies increased. However, IgM decreased slightly three weeks after the onset of symptoms. IgM and IgG antibody titers were also higher in people with severe infection than those with mild infection (11). Liu et al. examined the response pattern of anti-SARS-CoV-2 IgG and IgM antibodies in COVID-19 patients; COVID-19 infection was detected using RT-PCR. This study showed that IgM increased faster than IgG and reached a peak. The IgM antibodies started to decrease three weeks after infection. In contrast, the IgG antibody response continued and was preserved in patients (12).

According to the present study, it can be suggested that the antibody remains high in a high percentage of patients up to five months after infection. A recent study showed that the anti-SARS-CoV-2 IgG test was suitable for assessing previous virus exposure, although it illustrated that the test results could be negative during the first weeks after infection (13). The results also showed that patients whose antibodies remained positive for the second time had a higher percentage of symptoms, such as myalgia and weakness at the time of infection. Our results illustrated that the IgG level remained high after five months in patients who experienced anosmia during infection. Therefore, it can be said that anosmia is one of the symptoms that appear in patients and indicates a high antibody titer remaining after five months. Hence, if anosmia appears as a symptom of the disease in patients, it can indicate high immunity with the production of more antibodies in patients, which is an excellent prognostic factor. A recent study showed that high anti-SARS-CoV-2 IgG antibody titers in patients with olfactory disorders could be considered a warning sign of the disease (14). Based on our results, $6.5 \%$ of the total patients were re-infected with COVID-19 six months later; thus, re-infection can occur in patients.

\subsection{Conclusions}

Based on the present study, the anti-SARS-CoV-2 IgG test may be appropriate for identifying suspected patients with a negative PCR test. The study further showed that anosmia is a good prognostic factor for indicating high immunity in COVID-19 patients. Therefore, the healthcare system should do rapid screening tests and isolation measures for patients with anosmia. Also, re-infection may occur in some patients.

\section{Footnotes}

Authors' Contribution: B. M. designed the study and critically reviewed the manuscript. F. Z. and SB collected the clinical data. A. K. was the major contributor to writing the 


\begin{tabular}{|c|c|c|c|c|}
\hline & \multicolumn{2}{|c|}{ IgG } & \multirow{2}{*}{ Total } & \multirow{2}{*}{ P-Value } \\
\hline & Positive & Negative & & \\
\hline Anosmia & & & & $0.0001^{b}$ \\
\hline Positive & $19(82.6)$ & $4(17.4)$ & $23(100.0)$ & \\
\hline Negative & $48(33.3)$ & $96(66.7)$ & $144(100.0)$ & \\
\hline Total & $67(40.1)$ & $100(59.9)$ & $167(100.0)$ & \\
\hline
\end{tabular}

${ }^{a}$ Values are expressed as No. (\%) unless otherwise indicated.

${ }^{\mathrm{b}}$ A P-value less than 0.05 was considered the significance level.

manuscript. A. J. did the PCR and Ig-G titer tests. K. R. analyzed and interpreted the data. All authors read and approved the final manuscript.

Conflict of Interests: There was no conflict of interest.

Ethical Approval: The Research Ethics Committee of KUMS approved the study according to the criteria set by the Declaration of Helsinki (IR.MUK.REC.199.272).

Funding/Support: The Kurdistan University of Medical Sciences funded the study.

Informed Consent: Informed consent was obtained from all subjects.

\section{References}

1. Xu X, Chen P, Wang J, Feng J, Zhou H, Li X, et al. Evolution of the novel coronavirus from the ongoing Wuhan outbreak and modeling of its spike protein for risk of human transmission. Sci China Life Sci. 2020;63(3):457-60. doi: 10.1007/s11427-020-1637-5. [PubMed: 32009228]. [PubMed Central: PMC7089049].

2. Fan E, Beitler JR, Brochard L, Calfee CS, Ferguson ND, Slutsky AS, et al. COVID-19-associated acute respiratory distress syndrome: is a different approach to management warranted? Lancet Respir Med. 2020;8(8):816-21. doi: 10.1016/s2213-2600(20)30304-0.

3. Ji W, Wang W, Zhao X, Zai J, Li X. Cross-species transmission of the newly identified coronavirus 2019-nCoV. J Med Virol. 2020;92(4):43340. doi: 10.1002/jmv.25682. [PubMed: 31967321]. [PubMed Central: PMC7138088].

4. Riou J, Althaus CL. Pattern of early human-to-human transmission of Wuhan 2019 novel coronavirus (2019-nCoV), December 2019 to January 2020. Euro Surveill. 2020;25(4). doi: 10.2807/15607917.ES.2020.25.4.2000058. [PubMed: 32019669]. [PubMed Central: PMC7001239].

5. Challen R, Brooks-Pollock E, Read JM, Dyson L, Tsaneva-Atanasova $\mathrm{K}$, Danon L. Risk of mortality in patients infected with SARSCoV-2 variant of concern 202012/1: matched cohort study. BMJ. 2021;372:n579. doi: 10.1136/bmj.n579. [PubMed: 33687922]. [PubMed Central: PMC7941603].
6. Gombos K, Foldi M, Kiss S, Herczeg R, Gyenesei A, Geiger L, et al. Analysis of COVID-19-related RT-qPCR test results in Hungary: Epidemiology, diagnostics, and clinical outcome. Front Med (Lausanne). 2020;7. doi: 10.3389/fmed.2020.625673. [PubMed: 33575263]. [PubMed Central: PMC7870862].

7. Zhang J, Zhang X, Liu J, Ban Y, Li N, Wu Y, et al. Serological detection of 2019-nCoV respond to the epidemic: A useful complement to nucleic acid testing. Int Immunopharmacol. 2020;88. doi: 10.1016/j.intimp.2020.106861. [PubMed: 32771946]. [PubMed Central: PMC7391978].

8. Qin X, Shen J, Dai E, Li H, Tang G, Zhang L, et al. The seroprevalence and kinetics of IgM and IgG in the progression of COVID-19. BMC Immunol. 2021;22(1):14. doi: 10.1186/s12865-021-00404-0. [PubMed: 33596826]. [PubMed Central: PMC7887538].

9. Adams ER, Ainsworth M, Anand R, Andersson MI, Auckland K, Baillie JK, et al. Evaluation of antibody testing for SARS-CoV-2 using ELISA and lateral flow immunoassays. medRxiv. 2020;5:139. doi: 10.12688/wellcomeopenres.15927.1.[PubMed:33748431]. [PubMed Central: PMC7941096].

10. Hou H, Wang T, Zhang B, Luo Y, Mao L, Wang F, et al. Detection of IgM and IgG antibodies in patients with coronavirus disease 2019. Clin Transl Immunology. 2020;9(5). e01136. doi: 10.1002/cti2.1136. [PubMed: 32382418]. [PubMed Central: PMC7202656].

11. Long QX, Liu BZ, Deng HJ, Wu GC, Deng K, Chen YK, et al. Antibody responses to SARS-CoV-2 in patients with COVID-19. Nat Med. 2020;26(6):845-8. doi: 10.1038/s41591-020-0897-1. [PubMed: 32350462].

12. Liu X, Wang J, Xu X, Liao G, Chen Y, Hu CH. Patterns of IgG and IgM antibody response in COVID-19 patients. Emerg Microbes Infect. 2020;9(1):1269-74. doi: 10.1080/22221751.2020.1773324. [PubMed: 32515684]. [PubMed Central: PMC7448841].

13. Hoffman T, Nissen K, Krambrich J, Ronnberg B, Akaberi D, Esmaeilzadeh $\mathrm{M}$, et al. Evaluation of a COVID-19 IgM and IgG rapid test; an efficient tool for assessment of past exposure to SARS-CoV-2. Infect Ecol Epidemiol. 2020;10(1):1754538. doi: 10.1080/20008686.2020.1754538. [PubMed: 32363011]. [PubMed Central: PMC7178815].

14. Taziki Balajelini MH, Vakili MA, Saeidi M, Tabarraei A, Hosseini SM. Using anti-SARS-CoV-2 IgG and IgM antibodies to detect outpatient cases with olfactory and taste disorders suspected as mild form of COVID-19: A retrospective survey. SN Compr Clin Med. 2020:1-7. doi: 10.1007/s42399-020-00623-3. [PubMed: 33169109]. [PubMed Central: PMC7608292]. 